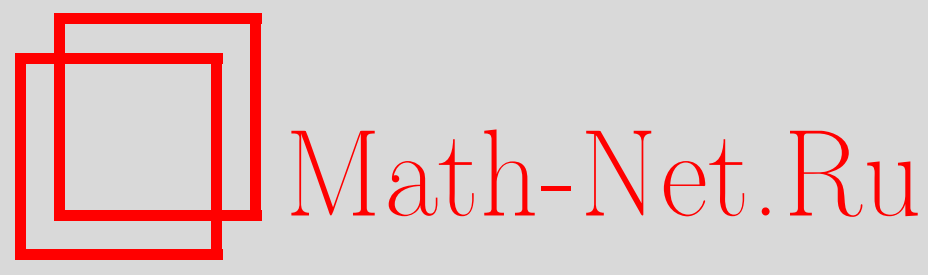

В. М. Трутнев, Плотность полиномиальных элементов в инвариантных подпространствах пространств целых функций экспоненциального типа, Матем. заметки, 2004, том 75, выпуск 3, 421-424

DOI: https://doi.org/10.4213/mzm45

Использование Общероссийского математического портала Math-Net.Ru подразумевает, что вы прочитали и согласны с пользовательским соглашением http://www.mathnet.ru/rus/agreement

Параметры загрузки:

IP : 3.85 .7 .115

26 апреля 2023 г., 15:26:31

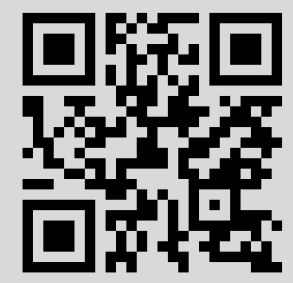




\title{
ПЛОТНОСТЬ ПОЛИНОМИАЛЬНЫХ ЭЛЕМЕНТОВ В ИНВАРИАНТНЫХ ПОДПРОСТРАНСТВАХ ПРОСТРАНСТВ ЦЕЛЫХ ФУНКЦИЙ ЭКСПОНЕНЦИАЛЬНОГО ТИПА
}

\section{B. М. Трутнев}

\begin{abstract}
Рассматриваются инвариантные относительно сдвига подпространства в пространстве целых функций экспоненциального типа многих комплексных переменных и приводятся условия, при которых множество экспоненциальных полиномов плотно в этих подпространствах. В частности, получены необходимые и достаточные условия плотности полиномиальных элементов.

Библиография: 10 названий.
\end{abstract}

Б. Мальгранж [1], исследуя вопрос об аппроксимации произвольных решений (в различных классах функций) полиномиальными решениями для дифференциального уравнения с постоянными коэффициентами, нашел необходимое и достаточное условие для такой апшроксимации: все неприводимые компоненты характеристического многочлена дифференциального уравнения должны обращаться в нуль в начале координат. Позднее В. П. Паламодов [2, гл. VII, $\S 8$, теорема 5] для систем дифференциальных уравнений доказал, что всякое решение может быть аппроксимировано экспоненциальными полиномами вида $p(z) \exp (z, \zeta), \zeta \in B$, где множество $B \supset \mathbb{C}^{n}$ имеет непустое пересечение с каждым многообразием из соответствующего набора нетеровских операторов и многообразий. Отсюда следует, что если все многообразия содержат начало координат, то каждое решение аппроксимируется полиномиальными решениями.

Многие результаты о решениях дифференциальных уравнений допускают распространение на случай уравнений свертки (см., например, обзоры [3], [4]) или даже на инвариантные относительно сдвига или дифференцирования подпространства в различных пространствах функций.

Целью настоящей статьи является распространение на инвариантные подпространства в рассмотренном ниже пространстве целых функций экспоненциального типа результатов Мальгранжа и Паламодова. Выбор этого класса пространств объясняется тем, что в них любое инвариантное подпространство допускает спектральный анализ и синтез (В. Н. Филиппов [5]) и, следовательно, отсутствуют известные контрпримеры Д. И. Гуревича [6].

Пусть $G$ - область Рунге в пространстве $\mathbb{C}^{n}, \mathscr{O}(G)$ - пространство функций, голоморфных в области $G$ с естественной топологией. Через $\mathscr{O}^{\prime}(G)$ обозначим сопряженное пространство к $\mathscr{O}(G)$, т.е. пространство линейных непрерывных функционалов на $\mathscr{O}(G)$, наделенное сильной топологией.

Пусть $L$ - отображение, которое всякому линейному функционалу $T \in \mathscr{O}^{\prime}(G)$ ставит в соответствие целую функцию экспоненциального роста $\widehat{T}(z)=T_{t}(\exp (t, z)), z \in \mathbb{C}^{n}$,

Работа выполнена при поддержке Российского фонда фундаментальных исследований, грант № 02-01-00167. 
где $(z, t)=z_{1} t_{1}+\cdots+z_{n} t_{n}$. Функция $\widehat{T}(z)$ называется преобразованием Лапласа функиионала $T$. Если образ $P_{G}$ пространства $\mathscr{O}^{\prime}(G)$ при отображении $L$ наделить индуцированной топологией, то отображение $L$ будет топологическим изоморфизмом (см., например, [7]).

Отображение $L$ и пространство $P_{G}$ хорошо известны, так же как и специальный принцип двойственности (см., например, [8]): между совокупностью $V$ всех подпространств в $P_{G}$, инвариантных относительно сдвига, и совокупностью всех замкнутых идеалов $V^{*}$ из $P_{G}^{\prime}$ можно установить взаимно однозначное соответствие по правилу: $V \longleftrightarrow V^{*}$ тогда и только тогда, когда $V^{*}=\operatorname{An} V$ и $V=\operatorname{An} V^{*}$. Под An $V$ понимается совокупность всех функционалов, обращающихся в нуль на $V$, а под An $V^{*}$ - совокупность всех функций из $P_{G}$, на которьх каждый функционал из $V^{*}$ обращается в нуль. Обозначим через $L^{*}$ сопряженное к $L$ отображение $L^{*}: P_{G}^{\prime} \rightarrow \mathscr{O}^{\prime \prime}(G)$ и через $\theta$ - канонический изоморфизм пространства $\mathscr{O}(G)$ на его второе сопряженное $\mathscr{O}^{\prime \prime}(G)$. Рассмотрим топологический изоморфизм $\chi=\theta^{-1} \circ L^{*}: P_{G}^{\prime} \rightarrow \mathscr{O}(G)$, при котором всякому инвариантному подпространству $V \subset P_{G}$ будет соответствовать замкнутьй идеал $I \subset \mathscr{O}(G), I=\chi(\operatorname{An} V)$.

Всякий элемент $F$ из $V$ является преобразованием Лапласа функционала $T$, ортогонального идеалу $I \subset \mathscr{O}(G)$. Идеал $I$ назьвается аннуляторным идеалом инвариант-

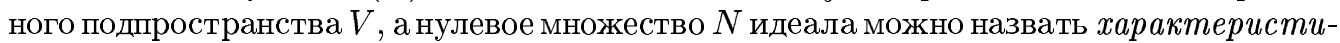
ческим аналитическим множеством инвариантного подпространства $V$.

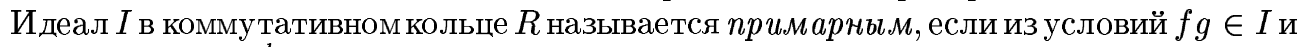
$g \notin I$ следует, что $f^{k} \in I$ при некотором натуральном $k$. Если кольцо $R$ нетерово, то всякий идеал представим в виде конечного пересечения примарных идеалов. Этот факт существенно используется в результатах Мальгранжа и Паламодова. Кольцо $\mathscr{O}(G)$, если множество $G$ открыто, не является нетеровым. Тем не менее в кольце $\mathscr{O}(G)$ существует аналогичное разложение, но, вообще говоря, счетное.

Семейство $\left\{N_{\alpha}\right\}_{\alpha \in A}$ подмножеств топологического пространства $X$ назьвается локально-конечным, если $\forall x \in X$ сушествует окрестность $U$ такая, что $U \cap N_{\alpha} \neq \varnothing$ лишь для конечного числа $\alpha \in A$. Семейство идеалов $\left\{I_{\alpha}\right\}_{\alpha \in A}$ в алгебре $\mathscr{O}(G)$ называется локально-конечньп, если семейство их нулевых множеств $N_{\alpha}=N\left(I_{\alpha}\right)$ локально-конечно в $G$.

Пусть идеал $J$ представим в виде

$$
J=\bigcap_{\alpha \in A} I_{\alpha} .
$$

Такоепредставление идеала $J$ называется несократимым разложениемна идеалы $I_{\alpha}$, если для всякого подмножества $B \varsubsetneqq A$

$$
J \neq \bigcap_{\beta \in B} I_{\beta} .
$$

Цитируемое ниже предложение доказано для более широкого класса пространств Штейна (см. [9], [10]).

ПРЕДЛОЖЕНИЕ. Всякий замкнутый идеал $I \subset \mathscr{O}(G)$, әде $G$ - область голоморфности в $\mathbb{C}^{n}$, допускает локально-конечное, несократимое разложение

$$
I=\bigcap_{\alpha \in A} I_{\alpha}
$$

где $I_{\alpha}-$ замкнутые, примарные идеаль в $\mathscr{O}(G)$.

Разложение (1) идеала $I$ не является однозначно определенньм, но связанное с ним семейство аналитических множеств

$$
\left\{N_{\alpha}\right\}_{\alpha \in A}, \quad N_{\alpha}=N\left(I_{\alpha}\right),
$$


определяется однозначно, причем каждое множество $N_{\alpha}$ является неприводимьп аналитическим множеством в $G$ (см. [10]).

Рассмотрим произвольное инвариантное подпространство $V \subset P_{G}$ отличное от всего пространства $P_{G}$. Пусть $I \subset \mathscr{O}(G)$ - его аннуляторньй идеал и $N$-характеристическое аналитическое множество.

Обозначим через $E^{a}, a \in G$, множество всех экспоненциальных полиномов вида $p(z) \exp (a, z)$ в подпространстве $V$, где $p(z)$ - полином.

Сформулируем критерий плотности экспоненциальных полиномов в инвариантных подпространствах.

Теорема 1. Пусть $V \subset P_{G}-$ инвариантное относительно сдвига подпространство, $I \subset \mathscr{O}(G)$ - его аннуляторный идеал, для которого выбрано некоторое примарное разлохсение (1), $u E=\bigcup_{a \in B} E^{a}$, әде $B \subset G$, - некоторое множество. Для того чтобы замыкание линейной оболочки множества $E$ совпадало с подпространством $V$, необходимо и достаточно, чтобы пересечение $B \cap N_{\alpha}$ было не пусто при любом $N_{\alpha}$ из ассочиированного набора неприводимых аналитических множеств (2).

ДокАЗАТЕЛЬСтво. Достаточность. Чтобы доказать, что замыкание линейной оболочки множества $E$ совпадает с подпространством $V$, достаточно доказать, что всякий функционал $S \in P_{G}^{\prime}$, равный нулю на $E$, обращается в нуль на $V$, т.е. что $S \in \operatorname{An} V$. Если рассмотреть $f=\chi(S) \in \mathscr{O}(G)$, то нам необходимо установить, что $f$ принадлежит аннуляторному замкнутому идеалу $I=\chi($ An $V)$. Обозначим через $\mathscr{O}_{a}$ кольцо ростков голоморфных функций в точке $a \in \mathbb{C}^{n}$, а через $I_{a}$ - идеал, порожденный идеалом $I$ в кольце $\mathscr{O}_{a}$. Имеется глубокая связь между локальньми идеалами $I_{a}$ и множествами $E^{a}$. А именно, справедливо следующее утверждение [5]: функиия $f \in \mathscr{O}(G)$ принадлежит идеалу $I_{a}$ тогда и только тогда, когда функиионал $\chi^{-1}(f) \in P_{G}^{\prime}$ ортогонален всем әлементам из $E^{a}$, и функиия $f$ принадлежст идеалу I тогда и только тогда, когда функиионал $\chi^{-1}(f) \in P_{G}^{\prime}$ ортогонален всем әлементам из $\bigcup_{a \in G} E^{a}$.

Очевидно из этого результата следует, что множество $E^{a}$ не пусто тогда и только тогда, когда идеал $I_{a} \neq \mathscr{O}_{a}$, т.е. точка $a$ принадлежит аналитическому множеству $N$. В силу этого остается доказать, что росток голоморфной функции $f \in \mathscr{O}(G)$ принадлежит всем локальным идеалам $I_{a}$, если точка $a$ принадлежит аналитическому множеству $N$. Итак, пусть $a \in N$. Аналитическое множество $N$ идеала $I$ представляется в окрестности данной точки в виде объединения конечного числа $N_{1}, \ldots, N_{l}$ неприводимых аналитических множеств из ассоциированного набора неприводимых аналитических множеств $(2)$, а идеал $I_{a}$ представляется в виде пересечения соответствующих идеалов $I_{1 a}, \ldots, I_{l a}$, порожденных идеалами $I_{1}, \ldots, I_{l}$ в кольце $\mathscr{O}_{a}$. Каждый идеал $I_{k}$, $k=1, \ldots, l$, примарен в $\mathscr{O}(G)$, хотя идеалы $I_{k}$ не обязаны быть примарными в кольце $O_{a}$. Рассмотрим некоторьй идеал $I_{k}$. По условию существует точка $b \in B \bigcap N_{k}$ такая, что функционал $S$ ортогонален $E^{b}$, т.е. росток $f_{b} \in I_{b}$ и, следовательно, $f_{b} \in I_{k b}$.

Нижеследующая лемма справедлива для примарных идеалов в произвольных пространствах Штейна см. [9].

Лемма. Пусть $J$ - примарный идеал в алгебре $\mathscr{O}(G)$, функиия $f \in \mathscr{O}(G)$ и - точка нулевого мнохсества идеала $J$. Функиия $f \in J$ тогда и только тогда, когда росток $f_{b} \in J_{b}$.

Применяя данную лемму к примарному идеалу $I_{k}$, получаем, что росток $f_{a} \in I_{k a}$ для всех $k$. Тем самым достаточность доказана.

Необходимость. Пусть множество $B \in G$ выбрано так, что замыкание линейной оболочки множества $E=\bigcup_{a \in B} E^{a}$ совпадает с подпространством $V$, но в разложении (1) 
двойственного идеала $I$ существует примарньй идеал $I_{\alpha_{0}}$, нулевое множество $N_{\alpha_{0}}$ которого не пересекается с множеством $B$. Рассмотрим идеал

$$
J=\bigcap_{\alpha \in A \backslash\left\{\alpha_{0}\right\}} I_{\alpha} \supset I .
$$

Разложение идеала $I$ на примарные компоненты несократимо, поэтому $J \neq I$. Но тогда существует функция $f \in J$ такая, что $f \notin I$. Так как $B \bigcap V_{\alpha_{0}}$ пусто, то локальные идеалы $I_{\alpha_{0} a}=\mathscr{O}_{a}$ во всякой точке $a \in B$ и вьполняется равенство $I_{a}=J_{a}$. В силу условия $f \in J$ росток $f_{a} \in I_{a}$. В силу цитированного вьше утверждения функционал $S=\chi^{-1}(f) \in P_{G}^{\prime}$ ортогонален всем $E^{a}$, а в силу плотности множества $E$ в $V$ ортогонален и $V$, т.е. $S \in$ An $V$. Отсюда следует, что $f \in I$. Противоречие с выбором $f$. Теорема полностью доказана.

ЗАмЕЧАНИЕ. Все функции из множества $E^{0}$ являются полиномами, поэтому, выбирая в теореме 1 множество $B=\{0\}$, мы получаем критерий плотности полиномиальных элементов в инвариантных подпространствах.

Теорема 2. Пусть $V \subset P_{G}-$ инвариантное относительно сдвига подпространство, $I \subset \mathscr{O}(G)$ - двойственный инвариантному подпространству $V$ идеал, для которого выбрано некоторое примарное разлохсение (1), u $0 \in G$. Для того чтобы замыкание множества полиномиальных әлементов из $V$ совпадало с подпространством $V$, необходимо и достаточно, чтобы каждое аналитическое множество $N_{\alpha}$ из ассочиированного набора неприводимых аналитических мнохсеств (2) проходило через начало координат.

\section{СПИСОК ЦИТИРОВАННОЙ ЛИТЕРАТУРЫ}

[1] Malgrange B. Existence et appoximation des solutons des équations aux derivées partielles et des équations de convolution // Ann. Inst. Fourier, Grenoble. 1955-1956. V. 6. P. 1-271.

[2] Паламодов В. П. Линейные дифференциальные операторы с постоянными коэффициентами. М.: Наука, 1967.

[3] Кривошеев А. С., Напалков В. В. Комплексньй анализ и операторы свертки // УМН. 1992. T. 47. №6. С. 3-58.

[4] Беренстейн К., Струппа Д. Комплексный анализ и уравнения в свертках // Итоги науки и технини. Современные проблемы математики. Фундамент. направления. Т. 54. М.: ВИНИТИ, 1989. С. 5-111.

[5] Филиппов В. Н. Спектральный синтез в некоторых пространствах целых функций экспоненциального типа // Матем. заметки. 1981. Т. 30. № 4. С. 527-534.

[6] Гуревич Д. И. Контрпримеры к проблеме Л. Шварца // Функцион. анализ и его прилож. 1975. Т. 9. № 2. С. 29-35.

[7] Напалков В. В. Уравнения свертки в многомерных пространствах. М.: Наука, 1982.

[8] Напалков В. В. Подпространства целых функций экспоненциального типа, инвариантные относительно сдвига // Сиб. матем. ж. 1973. Т. 15. № 2. С. 427-436.

[9] Foster O. Primärzerlegung in Steinschen Algebren // Math. Ann. 1964. V. 154. P. 307-329.

[10] Yum-Tong Siu. Noether-Lasker decomposition of coherent analytic subsheaves // Trans. Amer. Math. Soc. 1969. V. 135. P. 375-385. 